\title{
Irinotecan monotherapy offers advantage over combination therapy with irinotecan plus cisplatin in second-line setting for treatment of advanced gastric cancer following failure of fluoropyrimidine-based regimens
}

\author{
MASARU OBA, KEISHO CHIN, YOSHIMASA KAWAZOE, KOICHI TAKAGI, MARIKO OGURA, EIJI SHINOZAKI, \\ MITSUKUNI SUENAGA, SATOSHI MATSUSAKA, NOBUYUKI MIZUNUMA and KIYOHIKO HATAKE
}

\author{
Gastrointestinal Group, Division of Medical Oncology, Cancer Institute Hospital \\ of the Japanese Foundation for Cancer Research, Tokyo, Japan
}

Received November 17, 2010; Accepted January 18, 2011

DOI: $10.3892 / \mathrm{ol} .2011 .242$

\begin{abstract}
The optimal regimen of chemotherapy for gastric cancer in a second-line setting remains to be clarified. The aim of this retrospective study was to evaluate the efficacy and safety of second-line irinotecan treatment. A total of 134 patients with gastric cancer who had received prior chemotherapy with fluoropyrimidine-based regimens were treated with irinotecan $\left(150 \mathrm{mg} / \mathrm{m}^{2}\right.$ on days 1 and 15) alone every 4 weeks (Arm I) or irinotecan $\left(70 \mathrm{mg} / \mathrm{m}^{2}\right.$ on days 1 and 15) plus cisplatin ( $80 \mathrm{mg} / \mathrm{m}^{2}$ on day 1$)$ every 4 weeks (Arm IP) between April, 2004 and March, 2009. Patient characteristics, response rate, progression-free survival, overall survival and safety were investigated. Of 134 patients with recurrent or unresectable gastric cancer, 92 were treated in Arm I and 42 patients in Arm IP. Overall response rate in Arm I was $8.1 \%$, compared with $20.0 \%$ in Arm IP $(\mathrm{P}=0.65)$. Median progression-free survival (Arm I vs. IP; 2.6 vs. 2.7 months, $\mathrm{P}=0.73$ ) and median overall survival (Arm I vs. IP; 9.8 vs. 8.0 months, $\mathrm{P}=0.67$ ) did not differ between the two treatment groups. Neutropenia, leukopenia and anorexia were the most common grade $3 / 4$ adverse events, occurring significantly more frequently in Arm IP than in Arm I $(\mathrm{P}<0.05)$. Irinotecan may be a key agent, and serial irinotecan monotherapy is more beneficial as compared to irinotecan plus cisplatin in the treatment of advanced gastric cancer in second-line settings. Irinotecan monotherapy is beneficial compared to irinotecan plus cisplatin in second-line settings for the treatment of advanced gastric cancer refractory to fluoropyrimidine-based regimens.
\end{abstract}

Correspondence to: Dr Masaru Oba, Division of Medical Oncology, Cancer Institute Hospital of the Japanese Foundation for Cancer Research, 3-8-31 Ariake, Koto-ku, Tokyo 135-8550, Japan

E-mail: masaru.oba@jfcr.or.jp

Key words: gastric cancer, irinotecan, cisplatin, second-line chemotherapy

\section{Introduction}

Gastric cancer (GC) is a common type of cancer in East Asia and the second leading cause of cancer death worldwide. Advances in medicine resulted in this entity being detected and treated much earlier than previously in Japan and Korea. However, the disease has often achieved an advanced stage prior to detection; subsequently, curative resection may no longer be an option. Systemic chemotherapy is an essential treatment modality for advanced GC, yielding improvements in quality of life and prolonged survival. A number of phase 3 trials and one meta-analysis showed that a 5-fluorouracil (FU)-based regimen improves both survival and symptoms compared with best supportive care (BSC) alone (1-3). Although results of certain randomized phase 3 trials showed no survival benefit for FU plus cisplatin (CDDP) as compared to FU alone (4), an FU-based regimen in combination with CDDP is the most commonly used first-line treatment for advanced GC (5-7). In the phase 3 SPIRITS trial conducted in Japan (8), advanced GC patients treated with combination chemotherapy consisting of S-1 plus CDDP in a first-line setting had an overall survival (OS) of 13 months as compared with 11 months in patients treated with S-1 alone [hazard ratio (HR) for death 0.77; 95\% CI, 0.61-0.98; $\mathrm{P}=0.04]$. Although a median OS of more than 1 year was achieved with the S-1 plus CDDP regimen, the median progression-free survival (PFS) of the first-line regimen was 6 months, and approximately $75 \%$ of the patients received second-line treatment. However, the impact of the second-line chemotherapy, which may have contributed to the favorable survival benefit, remains to be determined, and there is no standard regimen following the failure of first-line fluoropyrimidine-based treatment.

Irinotecan (CPT-11) and its active metabolite, SN-38, bind reversibly to the topoisomerase I-DNA complex and induce cancer cell death by preventing religation of single-strand DNA breaks. Irinotecan has shown anti-tumoral activity in gastrointestinal cancers and is commonly used in a secondline setting for metastatic colorectal cancer, either alone or in combination with other agents $(9,10)$. The activity of CPT-11 as 
a single agent at a dosage of $100 \mathrm{mg} / \mathrm{m}^{2}$ weekly or $150 \mathrm{mg} / \mathrm{m}^{2}$ bi-weekly in advanced GC was reported in 45 patients who had received previous chemotherapy, and the overall response rate (RR) was $16.1 \%$ (11). Kanat et al reported that $350 \mathrm{mg} / \mathrm{m}^{2}$ tri-weekly CPT-11 in 16 patients in whom FU-based therapy was unsuccessful resulted in an RR of $12.5 \%$ and a median OS of 5 months (12). On the other hand, a number of small phase 2 trials investigated combination chemotherapy with CPT-11 and CDDP following the failure of prior chemotherapy. In a Japanese trial, CPT-11 (70 mg/m² on days 1 and 15) plus CDDP $\left(80 \mathrm{mg} / \mathrm{m}^{2}\right.$ on day 1$)$ every 4 weeks in 15 patients previously treated with chemotherapy yielded a response rate of $27 \%$ and significant toxicity (13). A Korean trial using the same combination regimen showed a median PFS and OS of 2 and 7.5 months, respectively, for the second-line group of 20 patients with metastatic or recurrent GC (14). Ajani et al administered CPT-11 $50 \mathrm{mg} / \mathrm{m}^{2}$ plus CDDP $30 \mathrm{mg} / \mathrm{m}^{2}$ weekly for 4 weeks over a 6-week cycle and achieved an RR of $31 \%$ and PFS of 7 weeks (15). However, severe toxic effects developed in the majority of patients, and a modification in dose was suggested. The aim of this retrospective study was to evaluate the efficacy and safety of CPT-11 in a second-line setting for recurrent or unresectable GC following the failure of fluoropyrimidine-based regimens.

\section{Patients and methods}

Patients. Second-line chemotherapy with a CPT-11-based regimen was administered in a total of 134 patients at the Cancer Institute Hospital of the Japanese Foundation for Cancer Research, Japan between April, 2004 and March, 2009. Of these patients, CPT-11 alone was administered in 92 patients who were CDDP-refractory or in whom adequate hydration proved difficult; a combination regimen of CPT-11 plus CDDP was administered in the remaining 42 patients, who were CDDP-naïve or sensitive. Patients were selected according to the following criteria: i) histologically confirmed gastric cancer with metastatic or recurrent disease; ii) an Eastern Cooperative Oncology Group (ECOG) performance status (PS) of 0 to 2; iii) failure of prior chemotherapy with a fluoropyrimidine-based regimen, either in an adjuvant setting or for metastatic disease; iv) no findings of peritoneal metastasis with radiologically confirmed intestinal stenosis or massive ascites; v) age $\geq 18$ years; vi) adequate function of bone marrow, liver and kidney; vii) no synchronous double cancer or other serious disease; and viii) availability of informed consent prior to the administration of treatment.

Treatment. In patients receiving CPT-11 alone, the regimen consisted of infusional $150 \mathrm{mg} / \mathrm{m}^{2} \mathrm{CPT}-11$ on days 1 and 15 every 4 weeks (Arm I). In patients receiving CPT-11 plus CDDP, the regimen consisted of infusional $70 \mathrm{mg} / \mathrm{m}^{2}$ CPT-11 on days 1 and 15 and $80 \mathrm{mg} / \mathrm{m}^{2} \mathrm{CDDP}$ by intravenous drip infusion on day 1 with adequate hydration, to be repeated every 4 weeks (Arm IP). After $500 \mathrm{mg} / \mathrm{m}^{2}$ CDDP was administered, CPT-11 alone was continued every 2 weeks. Patients were pre-medicated intravenously with $5-\mathrm{HT}_{3}$ blocker and dexamethasone. Chemotherapy was administered until disease progression, occurrence of intolerable toxicity, or withdrawal from treatment at the patient's request.
Response and toxicity evaluation. A CT scan was carried out every 2 cycles of therapy to document the extent of disease and evaluate the response to treatment. Objective responses in measurable lesions were evaluated according to the guidelines of the Response Evaluation Criteria in Solid Tumors Committee (RECIST 1.0). Symptomatic toxicities and laboratory data were monitored every 2 weeks at the outpatient department. Toxicities were evaluated according to the Common Toxicity Criteria for Adverse Events, version 3.0 (CTCAE v.3.0). Dose reduction and treatment delays were recommended according to the extent of hematological and non-hematological toxicities.

Statistical analysis. Progression-free survival was calculated from the first day of CPT-11 treatment until the time of the first occurrence of progression, death from any cause, or the date of last follow-up if none of the preceding events had occurred. Overall survival was calculated from the first day of CPT-11 treatment to the date the patient succumbed to the disease or the date of the last follow-up visit. Survival curves were obtained using the Kaplan-Meier method. Univariate analysis of PFS and OS was performed using the log-rank test. Correlations between independent factors, treatment, and PFS and OS were determined by multivariate analysis using the Cox proportional hazards regression model in each arm. Statistical analyses were performed using SPSS (SPSS Inc., IL, USA). P-values were two-sided, with $\mathrm{P}<0.05$ indicating statistical significance.

\section{Results}

Patient characteristics. The patients were evaluable for survival parameters and toxicity. Patient characteristics are shown in Table I. Median ages of patients were 61 years (range 19-82) in Arm I and 57.5 years (range 28-73) in Arm IP, and the majority of the study population was male (72.4\%). A total of 133 patients $(99.3 \%)$ had an ECOG PS of 0 or 1. Primary lesions were present in 41 of 92 patients (44.6\%) in Arm I and in 8 of 42 patients (19.0\%) in Arm IP. The patients had received prior chemotherapies with regimens containing S-1, S-1 plus CDDP, capecitabine plus CDDP, or 5-FU plus methotrexate. Prior treatment with S-1 was administered in an adjuvant setting in 10 of $92(10.9 \%)$ patients in Arm I and in 10 of 42 (23.8\%) patients in Arm IP. Tumor response was evaluated in $109(81.3 \%)$ patients with measurable target lesions. Lymph nodes $(42.5 \%)$, peritoneum $(39.6 \%)$, and liver (35.8\%) were the most common metastatic sites. Histological types were intestinal in 47 patients $(35.1 \%)$ and diffuse in 87 patients $(64.9 \%)$ according to the Lauren classification. A total of 72 of 92 (78.3\%) patients in Arm I, and 33 of 42 (78.6\%) patients in Arm IP, received chemotherapy in a third-line setting.

Response and survival. No complete response (CR) was observed in the 109 patients assessable for response; RR was 8.1\% in Arm I and 20\% in Arm IP. Disease control (partial response, PR; and stable disease, SD) rate was 54.1\% in Arm I and $54.3 \%$ in Arm IP. Median PFS was comparable between 2.6 months (95\% CI, 2.2-3.0) in Arm I and 2.7 months (95\% CI, 1.5-3.9) in Arm IP (P=0.73) (Fig. 1). Median OS was 9.8 (95\% CI, 7.8-11.8) months in Arm I and 8 months 


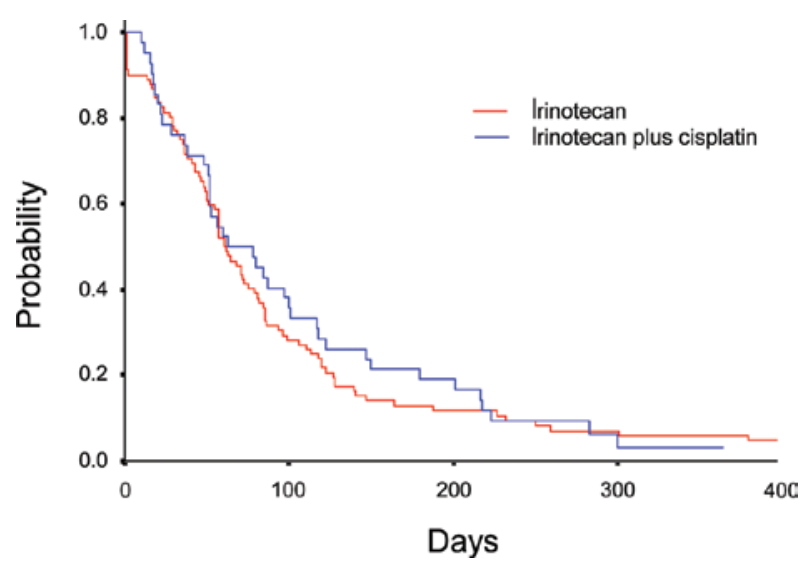

Figure 1. Progression-free survival (PFS) from the start of second-line chemotherapy.

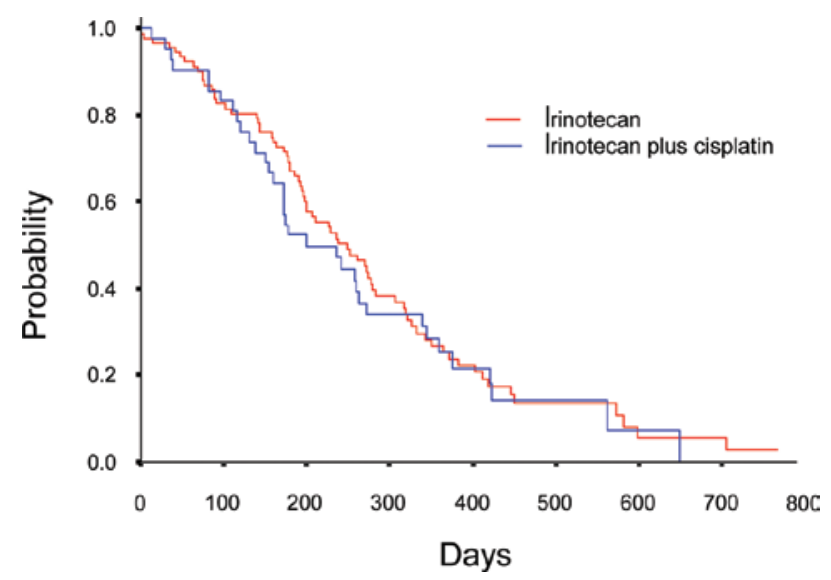

Figure 2. Overall survival (OS) from the start of second-line chemotherapy.

Table I. Patient characteristics $(n=134)$.

\begin{tabular}{|c|c|c|}
\hline Characteristics & $\begin{array}{c}\text { Arm I, n }(\%) \\
\quad(n=92)\end{array}$ & $\begin{array}{c}\text { Arm IP, n }(\%) \\
\quad(n=42)\end{array}$ \\
\hline \multicolumn{3}{|l|}{ Gender } \\
\hline Male & $67(72.8)$ & $30(71.4)$ \\
\hline Female & $25(27.2)$ & $12(28.6)$ \\
\hline Median age, (range) & $61(19-82)$ & $57.5(28-73)$ \\
\hline \multicolumn{3}{|l|}{ ECOG PS } \\
\hline 0 & $73(79.3)$ & $34(81.0)$ \\
\hline 1 & $18(19.6)$ & $8(19.0)$ \\
\hline 2 & $1 \quad(1.1)$ & $0 \quad(0.0)$ \\
\hline Previous gastrectomy (+) & $51(55.4)$ & $34(81.0)$ \\
\hline \multicolumn{3}{|l|}{ Histological type ${ }^{a}$} \\
\hline Intestinal & $37(40.2)$ & $10(23.8)$ \\
\hline Diffuse & $55(59.8)$ & $32(76.2)$ \\
\hline Target lesions (+) & $74(80.4)$ & $35(83.3)$ \\
\hline Peritoneal metastasis $(+)$ & $36(39.1)$ & $17(40.5)$ \\
\hline \multicolumn{3}{|l|}{ No. of involved organs } \\
\hline 1 & $42(45.7)$ & $19(45.2)$ \\
\hline$\geq 2$ & $50(54.3)$ & $23(54.8)$ \\
\hline \multicolumn{3}{|l|}{ Prior chemotherapy ${ }^{b}$} \\
\hline S-1 & $21(22.8)$ & $25(59.5)$ \\
\hline S-1 plus CDDP & $55(59.8)$ & $4 \quad(9.5)$ \\
\hline CAP plus CDDP & $4 \quad(4.3)$ & $0 \quad(0)$ \\
\hline 5-FU plus MTX & $2(2.2)$ & $1 \quad(2.4)$ \\
\hline Others & $0 \quad(0)$ & $2(4.8)$ \\
\hline Adjuvant S-1 & $10(10.9)$ & $10(23.8)$ \\
\hline Third-line chemotherapy (+) & $72(78.3)$ & $33(78.6)$ \\
\hline
\end{tabular}

aLauren classification. ${ }^{\mathrm{b} C D D P}$, cisplatin; CAP, capecitabine; 5-FU, fluorouracil; MTX, methotrexate.

(95\% CI, 5.1-11.0) in Arm IP ( $\mathrm{P}=0.67)$ (Fig. 2). No significant difference was observed between Arm I and Arm IP in either PFS or OS.
Prognostic factor analysis. Multivariate analysis was performed to assess the effects of age, adjuvant chemotherapy, degrees of response to first- and second-line chemotherapy, 
Table II. Frequency of grade 3/4 adverse events.

\begin{tabular}{lcr}
\hline Adverse events (AEs) & $\begin{array}{c}\text { Arm I, } \mathrm{n}(\%) \\
(\mathrm{n}=92)\end{array}$ & $\begin{array}{c}\text { Arm IP, } \mathrm{n}(\%) \\
(\mathrm{n}=42)\end{array}$ \\
\hline Hematological AEs & & \\
Leukopenia & $4(4.3)$ & $7(16.7)$ \\
Neutropenia & $7(7.6)$ & $22(52.4)$ \\
Anemia & $3(3.3)$ & $2(4.8)$ \\
Thrombocytopenia & $1(1.1)$ & $1(2.4)$ \\
Febrile neutropenia & $1(1.1)$ & $3(7.1)$ \\
Non-hematological AEs & & \\
Anorexia & $5(5.4)$ & $7(16.7)$ \\
Nausea & $2(2.2)$ & $3(7.1)$ \\
Fatigue & $1(1.1)$ & $2(4.8)$ \\
Diarrhea & $3(3.3)$ & $3(7.1)$ \\
Hyperkalemia & $0(0)$ & $1(2.4)$ \\
Increased transaminase & $0(0)$ & $1(2.4)$ \\
Treatment-related deaths & $1(1.1)$ & $0(0)$
\end{tabular}

histological type, prior gastrectomy, measurable target lesions, peritoneal metastasis and number of organs with metastasis at baseline. In Arm I, PFS was significantly longer in patients aged $<65$ years $(\mathrm{P}=0.049, \mathrm{HR}=0.45)$ and with non-peritoneal metastasis $(\mathrm{P}=0.026, \mathrm{HR}=0.46)$. In Arm IP, PFS was significantly longer in patients who received adjuvant chemotherapy $(\mathrm{P}=0.035, \mathrm{HR}=0.27)$. The results showed that OS in Arm I was significantly longer in patients in whom disease control was obtained with second-line treatment $(\mathrm{P}<0.001, \mathrm{HR}=0.39)$. Moreover, histologically, intestinal type was an independent prognostic factor for OS in Arm I ( $\mathrm{P}=0.009, \mathrm{HR}=0.45)$. Disease control with second-line treatment $(\mathrm{P}<0.001, \mathrm{HR}=0.13)$ and one organ showing metastasis $(\mathrm{P}=0.012, \mathrm{HR}=0.14)$ were independently correlated with a longer OS in Arm IP.

Adverse events. Adverse effects are shown in Table II. The most frequent grade $3 / 4$ adverse hematological event was neutropenia, which was observed in 7 of $92(7.6 \%)$ patients in Arm I and in 22 of 42 (52.4\%) patients in Arm IP $(\mathrm{P}<0.001)$. Leukopenia was observed in 4 of 92 (4.3\%) patients in Arm I and in 7 of $42(16.7 \%)$ patients in Arm IP $(\mathrm{P}<0.05)$. Septic shock and death within 30 days of the last administration of CPT-11 were observed in one patient (1.1\%) receiving CPT-11 alone. The most common grade $3 / 4$ adverse non-hematological event was anorexia, which was observed in 5 of $92(5.4 \%)$ patients in Arm I and in 7 of $42(16.7 \%)$ patients in Arm IP $(\mathrm{P}<0.05)$.

\section{Discussion}

Numerous studies have indicated a survival benefit for treatment of advanced GC in a first-line setting. However, although the SPIRITS trial demonstrated an OS of more than 1 year, PFS with S-1 plus CDDP in a first-line setting was only 6 months, and $74 \%$ of the patients received second-line chemotherapy (8). Moreover, in the JCOG9912 trial, OS and PFS were 11.4 and 4.2 months, respectively, in the S- 1 alone arm, and $74 \%$ of the patients received second-line chemotherapy (16). On the other hand, in the FLAGS global trial, OS and PFS were 8.6 and 4.8 months, respectively, in the S-1 plus CDDP arm, and only $31 \%$ of the patients received second-line chemotherapy (17). This suggests that in certain cases, optimal second-line chemotherapy contributes to the favorable OS observed with first-line treatment. However, few well-designed, randomized trials have been conducted for treatment in a second-line setting, and the optimal regimen following failure of first-line chemotherapy remains controversial. Recently, Thuss-Patience et al reported that CPT- 11 alone $\left(250 \mathrm{mg} / \mathrm{m}^{2}\right.$ every 3 weeks, to be increased to $350 \mathrm{mg} / \mathrm{m}^{2}$, depending on toxicity) as secondline treatment significantly improved OS compared to BSC $(\mathrm{HR}=0.48 ; 95 \% \mathrm{CI}, 0.25-0.92 ; \mathrm{P}=0.023)$ in patients previously treated with only one regimen (18). This suggests that CPT-11 is a key novel agent in the treatment of advanced GC in a secondline setting. In the current study, overall RR in Arm IP was $20 \%$, as compared with $8.1 \%$ in Arm I ( $P=0.65)$. The median PFS (Arm IP vs. I; 2.7 vs. 2.6 months) and median OS (Arm IP vs. I; 8 vs. 9.8 months) did not differ between the two treatment groups. Although this was a retrospective study, the results provide evidence that patients in whom fluoropyrimidinebased first-line chemotherapy is unsuccessful may derive a benefit from second-line treatment with CPT-11.

Multivariate analysis revealed that in both arms, patients with disease controlled by second-line CPT-11 treatment had a significantly longer OS. The proportion of patients who received third-line treatment in our study was $78.4 \%$, and taxane agents were administered in $94.3 \%$ of those patients. Third-line chemotherapy including taxane agents may also have contributed to the survival benefit. Patients with recurrent GC following adjuvant S-1 monotherapy had a longer PFS in Arm IP. Nagashima et al suggested that two or three favorable phenotypes, p53-negative, bcl-2-negative and VEGF-positive, are favorable predictors of therapeutic effects 
in patients treated with CPT-11 plus CDDP (19). Expression of specific chemosensitivity-related genes is currently being investigated in patients enrolled in the JCOG9912 trial. It has been suggested that certain CDDP-naïve populations with prior S-1 adjuvant treatment may benefit from a CPT-11 plus CDDP regimen.

Defining the optimal CPT-11 regimen from the results of the present study is difficult. However, the selection of the CDDP combination in a second-line setting appears to be unlikely, as no benefit was noted in terms of PFS or OS in Arm IP as compared to Arm I. Moreover, neutropenia, leukopenia and anorexia, the most common grade $3 / 4$ adverse events, occurred more frequently in Arm IP than in Arm I.

The results suggest that, in chemotherapy for advanced GC in a second-line setting, CPT-11 is a key novel agent and that serial CPT-11 monotherapy is beneficial as compared to CDDP combination therapy. Further prospective clinical trials may be useful in developing individualized optimal treatments, providing evidence concerning the efficacy of molecularly targeted agents and the utility of biological markers for the treatment of advanced GC in a second-line setting.

\section{References}

1. Murad AM, Santiago FF, Petroianu A, Rocha PR, Rodrigues MA and Rausch M: Modified therapy with 5-fluorouracil, doxorubicin, and methotrexate in advanced gastric cancer. Cancer 72 : 37-41, 1993.

2. Pyrhönen S, Kuitunen T, Nyandoto P and Kouri M: Randomised comparison of fluorouracil, epidoxorubicin and methotrexate (FEMTX) plus supportive care with supportive care alone in patients with non-resectable gastric cancer. Br J Cancer 71: 587-591, 1995

3. Wagner AD, Grothe W, Haerting J, Kleber G, Grothey A and Fleig WE: Chemotherapy in advanced gastric cancer: a systematic review and meta-analysis based on aggregate data. J Clin Oncol 24: 2903-2909, 2006.

4. Ohtsu A, Shimada Y, Shirao K, et al: Randomized phase III trial of fluorouracil alone versus fluorouracil plus cisplatin versus uracil and tegafur plus mitomycin in patients with unresectable, advanced gastric cancer: The Japan Clinical Oncology Group Study (JCOG9205). J Clin Oncol 21: 54-59, 2003.

5. Vanhoefer U, Rougier P, Wilke H, et al: Final results of a randomized phase III trial of sequential high-dose methotrexate, fluorouracil, and doxorubicin versus etoposide, leucovorin, and fluorouracil versus infusional fluorouracil and cisplatin in advanced gastric cancer: A trial of the European Organization for Research and Treatment of Cancer Gastrointestinal Tract Cancer Cooperative Group. J Clin Oncol 18: 2648-2657, 2000
6. Van Cutsem E, Moiseyenko VM, Tjulandin S, et al: Phase III study of docetaxel and cisplatin plus fluorouracil compared with cisplatin and fluorouracil as first-line therapy for advanced gastric cancer: a report of the V325 Study Group. J Clin Oncol 24: 4991-4997, 2006.

7. Cunningham D, Starling N, Rao S, et al: Capecitabine and oxaliplatin for advanced esophagogastric cancer. N Engl J Med 358: 36-46, 2008.

8. Koizumi W, Narahara H, Hara T, et al: S-1 plus cisplatin versus S-1 alone for first-line treatment of advanced gastric cancer (SPIRITS trial): a phase III trial. Lancet Oncol 9: 215-221, 2008.

9. Rougier P, van Cutsem E, Bajetta E, et al: Randomised trial of irinotecan versus fluorouracil by continuous infusion after fluorouracil failure in patients with metastatic colorectal cancer. Lancet 352: 1407-1412, 1998

10. Cunningham D, Pyrhönen S, James RD, et al: Randomised trial of irinotecan plus supportive care versus supportive care alone after fluorouracil failure for patients with metastatic colorectal cancer. Lancet 352: 1413-1418, 1998.

11. Futatsuki K, Wakui A, Nakao I, et al: Late phase II study of irinotecan hydrochloride (CPT-11) in advanced gastric cancer. CPT-11 Gastrointestinal Cancer Study Group. Gan To Kagaku Ryoho 21: 1033-1038, 1994.

12. Kanat O, Evrensel T, Manavoglu O, et al: Single-agent irinotecan as second-line treatment for advanced gastric cancer. Tumori 89 : 405-407, 2003

13. Boku N, Ohtsu A, Shimada Y, et al: Phase II study of a combination of irinotecan and cisplatin against metastatic gastric cancer. J Clin Oncol 17: 319-323, 1999.

14. Im CK, Rha SY, Jeung HC, et al: A phase II study of a combined biweekly irinotecan and monthly cisplatin treatment for metastatic or recurrent gastric cancer. Am J Clin Oncol 33: 56-60, 2010.

15. Ajani JA, Baker J, Pisters PW, et al: Irinotecan/cisplatin in advanced, treated gastric or gastroesophageal junction carcinoma. Oncology 16: 16-18, 2002.

16. Boku N, Yamamoto S, Fukuda H, et al: Fluorouracil versus combination of irinotecan plus cisplatin versus S-1 in metastatic gastric cancer: a randomised phase 3 study. Lancet Oncol 10: 1063-1069, 2009

17. Ajani JA, Rodriguez W, Bodoky G, et al: Multicenter phase III comparison of cisplatin/S-1 with cisplatin/infusional fluorouracil in advanced gastric or gastroesophageal adenocarcinoma study: the FLAGS trial. J Clin Oncol 28: 1547-1553, 2010.

18. Thuss-Patience PC, Kretzschmar A, Deist T, et al: Irinotecan versus best supportive care (BSC) as second-line therapy in gastric cancer. A randomized phase III study of the Arbeitsgemeinschaft Internistische Onkologie (AIO). J Clin Oncol 27 (Suppl 15): 4540, 2009.

19. Nagashima F, Boku N, Ohtsu A, et al: Biological markers as a predictor for response and prognosis of unresectable gastric cancer patients treated with irinotecan and cisplatin. Jpn J Clin Oncol 35: 714-719, 2005. 